\title{
Correction to: Diverse, evolving conformer populations drive distinct phenotypes in frontotemporal lobar degeneration caused by the same MAPT-P301L mutation
}

\author{
Nathalie Daude ${ }^{1} \cdot$ Chae Kim $^{2}$ - Sang-Gyun Kang ${ }^{1}$. Ghazaleh Eskandari-Sedighi ${ }^{1,7} \cdot$ Tracy Haldiman $^{2}$ - Jing Yang ${ }^{1}$. \\ Shelaine C. Fleck ${ }^{1,7}$. Erik Gomez-Cardona ${ }^{7}$. Zhuang Zhuang Han ${ }^{1,7}$. Sergi Borrego-Ecija ${ }^{4}$. Serene Wohlgemuth ${ }^{1}$. \\ Olivier Julien ${ }^{7}$. Holger Wille ${ }^{1,7} \cdot$ Laura Molina-Porcel $^{5} \cdot$ Ellen Gelpi $^{4,6} \cdot$ Jiri G. Safar ${ }^{2,3}$ • David Westaway ${ }^{1,7}$
}

Published online: 30 January 2021

(c) Springer-Verlag GmbH Germany, part of Springer Nature 2021

\section{Correction to: Acta Neuropathologica (2020) 139:1045-1070 \\ https://doi.org/10.1007/s00401-020-02148-4}

In the original publication, Table 1 column heads are incorrectly formatted and aligned. The corrected Table 1 is given here.
Publisher's Note Springer Nature remains neutral with regard to jurisdictional claims in published maps and institutional affiliations.

The original article can be found online at https://doi.org/10.1007/ s00401-020-02148-4.

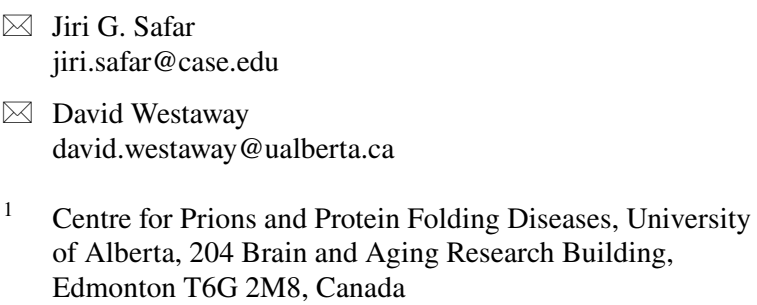

1 Centre for Prions and Protein Folding Diseases, University of Alberta, 204 Brain and Aging Research Building, Edmonton T6G 2M8, Canada

2 Department of Pathology, Case Western Reserve University, Institute of Pathology Building, Rm 406, 2085 Adelbert Road, Cleveland, OH 44106-4907, USA

3 Department of Neurology, Case Western Reserve University, Institute of Pathology Building, Rm 406, 2085 Adelbert Road, Cleveland, OH 44106-4907, USA
4 Neurological Tissue Bank of the Biobanc, Hospital Clinic, IDIBAPS, Barcelona, Spain

5 Neurology Department, Hospital Clinic, IDIBAPS, Barcelona, Spain

6 Division of Neuropathology and Neurochemistry, Department of Neurology, Medical University of Vienna, Vienna, Austria

7 Department of Biochemistry, University of Alberta, Edmonton, AB, Canada 


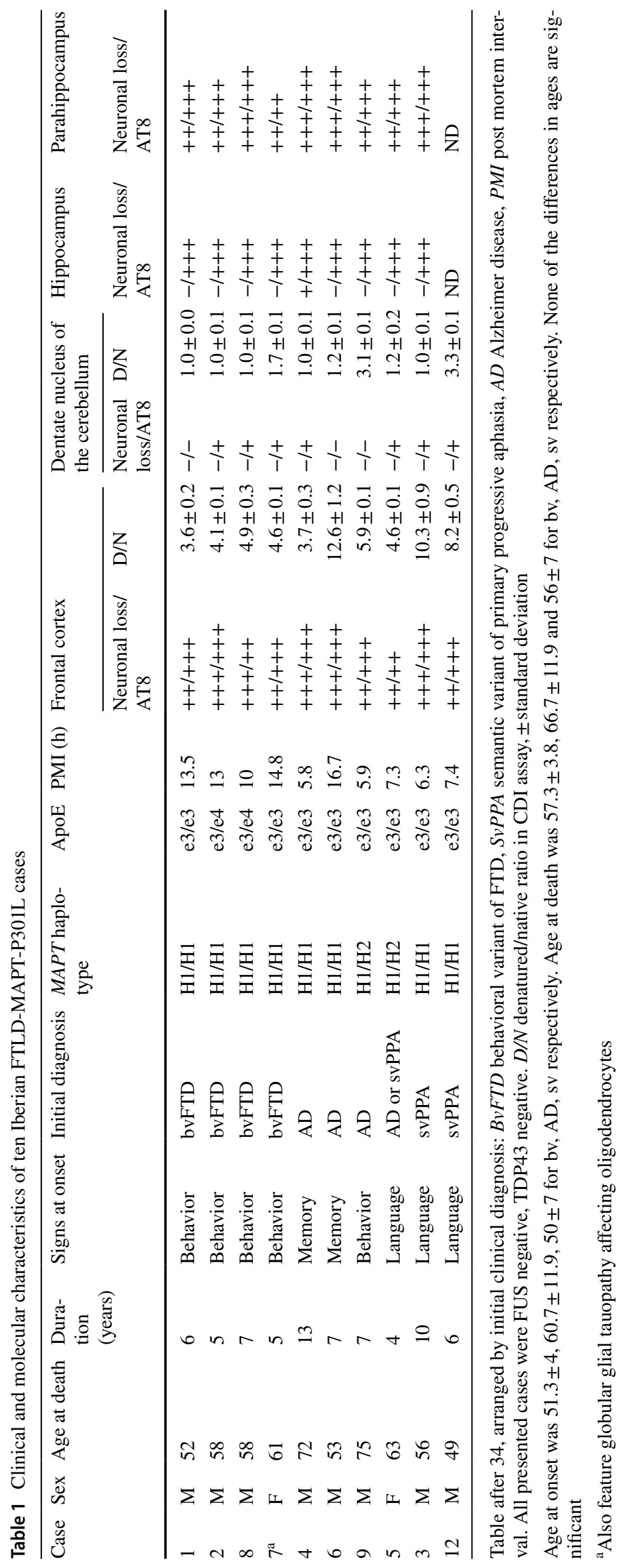

